\title{
Analysis and Control of Nonlinear Actuator Dynamics Based on the Sum of Squares Programming Method
}

\author{
Balázs Németh and Péter Gáspár
}

\begin{abstract}
The paper analyses the reachability characteristics of the brake system in order to determine its abilities for the entire vehicle system. The method is based on the nonlinear polynomial Sum-of-Squares programming method, in which the shape of the reachable set of the brake is calculated. Since the method can be applied to other actuators, a theory-based structure can be built for the coordination and reconfiguration of the actuators. The method is illustrated at various velocities and road conditions.
\end{abstract}

\section{INTRODUCTION AND MOTIVATION}

Various theoretical methods have already been proposed for the analysis of actuators. Comprehensive studies on both system and structural stability analyses of the vehicle model were presented in [1]. The design of lateral stability control based on set-theoretical methods was proposed by [2]. In another method the uncertain effects of the driver were also considered, see [3]. A large operating region accessible by the driver and smooth interventions at the stability boundaries were proposed by [4], [5]. A reachable set-based analysis was presented to illustrate the benefits of steering and suspension controller integration in [6].

Although the reachable set analysis of linear vehicle models can be a relatively fast and easily applicable technique for actuator intervention limit determination, it has some drawbacks. In the paper a nonlinear polynomial Sum-ofSquares (SOS) programming method is applied to calculate the shape of the reachable sets of actuators. In the paper the reachable set analysis focuses on the brake. Since the method can also be applied to other actuators, a theory-based structure can be built for the coordination and reconfiguration of the actuators.

The paper is organized as follows. In Section II the nonlinear polynomial vehicle model and stability regions are formulated. The fundamentals of the used SOS programming method are detailed in Section III. In Section IV the computation method of maximum Controlled Invariant Sets of polynomial lateral dynamical vehicle model is presented. In Section V control strategies using Controlled Invariant Sets are presented both in linear and nonlinear regions. In Section VI the operation of the control strategy is demonstrated through a simulation example. Section VII contains some concluding remarks.

The research has been conducted as part of the project TÁMOP-4.2.2.A11/1/KONV-2012-0012: Basic research for the development of hybrid and electric vehicles. The Project is supported by the Hungarian Government and co-financed by the European Social Fund.

The authors are with Systems and Control Laboratory, Institute for Computer Science and Control, Hungarian Academy of Sciences, Kende u. 13-17, H-1111 Budapest, Hungary. E-mail: [nemeth.balazs; gaspar.peter] Qsztaki.mta.hu

\section{NONLINEAR MODELING OF LATERAL VEHICLE DYNAMICS}

In the section the nonlinear modeling of lateral vehicle dynamics is presented. In the model the nonlinearities of the tire characteristics are taken into consideration. The vehicle dynamics is formed in two equations, i.e., the lateral force and the moment of the yaw dynamics, in which the control input is the differential braking moment:

$$
\begin{aligned}
J \ddot{\psi} & =\mathcal{F}_{1}\left(\alpha_{1}\right) l_{1}-\mathcal{F}_{2}\left(\alpha_{2}\right) l_{2}+M_{b r} \\
m v(\dot{\psi}+\dot{\beta}) & =\mathcal{F}_{1}\left(\alpha_{1}\right)+\mathcal{F}_{2}\left(\alpha_{2}\right)
\end{aligned}
$$

where $m$ is the mass of the vehicle, $J$ is yaw-inertia, $l_{1}$ and $l_{2}$ are geometric parameters. $\beta$ is the side-slip angle of the chassis, $\dot{\psi}$ is yaw-rate and $M_{b r}$ is the differential braking moment. $\mathcal{F}_{1}\left(\alpha_{1}\right)$ and $\mathcal{F}_{2}\left(\alpha_{2}\right)$ represent lateral tire forces, which depend on tire side-slip angles $\alpha_{1}$ and $\alpha_{2}$.

Several tire models have been published, see e.g., [7], [8]. In the paper a polynomial tire modeling approach is presented, by which the nonlinearities of the tire characteristics are considered in a given operation range. The nonlinear characteristics of lateral tire force in the function of tire side-slip $\alpha$ are illustrated in Figure 1. The polynomial approximation is formulated as:

$$
\mathcal{F}(\alpha)=\sum_{k=1}^{k=n} c_{k} \alpha^{k}=c_{1} \alpha+c_{2} \alpha^{2}+\ldots+c_{n} \alpha^{n}
$$

In the proposed method exponent $n$ is chosen 10 , in which the approximation the tire model is valid between $\alpha=$ $-12^{\circ} \ldots+12^{\circ}$.

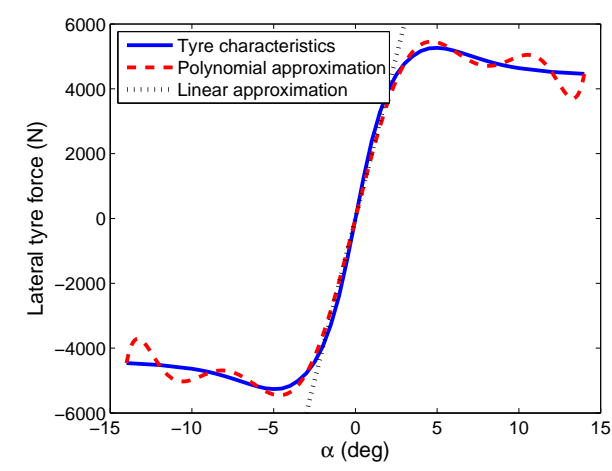

Fig. 1. Modeling of lateral tire force

At stable driving conditions the relationships between the tire side-slip angles for the front and rear axles, the steering 
angle of the vehicle and the side-slip angle of the chassis can be approximated as follows: $\alpha_{1}=\delta-\beta-\frac{\dot{\psi} l_{1}}{v}, \alpha_{2}=$ $-\beta+\frac{\dot{\psi} l_{2}}{v}$. Consequently, the yaw-rate and side-slip of the vehicle can be expressed in the following forms:

$$
\dot{\psi}=v \frac{\alpha_{2}-\alpha_{1}+\delta}{l_{1}+l_{2}}, \quad \beta=-\frac{\alpha_{1} l_{2}+\alpha_{2} l_{1}-l_{2} \delta}{l_{1}+l_{2}} .
$$

Now the vehicle model is reformulated:

$$
\begin{aligned}
\dot{\alpha}_{2}-\dot{\alpha}_{1}= & {\left[\frac{l_{1}+l_{2}}{J v}\left(\mathcal{F}_{1}\left(\alpha_{1}\right) l_{1}-\mathcal{F}_{2}\left(\alpha_{2}\right) l_{2}\right)\right]-} \\
& -\dot{\delta}+\frac{l_{1}+l_{2}}{J v} M_{b r} \\
\dot{\alpha}_{1} l_{2}+\dot{\alpha}_{2} l_{1}= & v\left(\alpha_{2}-\alpha_{1}\right)-\frac{l_{1}+l_{2}}{m v}\left[\mathcal{F}_{1}\left(\alpha_{1}\right)+\mathcal{F}_{2}\left(\alpha_{2}\right)\right]+ \\
& +v \delta+l_{2} \dot{\delta}
\end{aligned}
$$

The rearrangement of the vehicle model shows that the new states of the model are tire slip angles $\alpha_{1}$ and $\alpha_{2}$. In this way the nonlinearity of the lateral tire forces $\mathcal{F}_{1}, \mathcal{F}_{2}$ can be considered. However, (5) includes the time-derivative of the front-wheel steering angle. Since $\delta$ is a control input, $\dot{\delta}$ is modeled as below: $\dot{\delta} \cong \max (|\dot{\delta}| /|\delta|) \cdot \delta=\nu \cdot \delta$, where parameter $\nu$ represents the relationship between the maximum steering value and the variation speed of $\delta$.

Introducing the following notations

$$
\begin{aligned}
\mathrm{f}_{1}= & \frac{l_{1}}{J v}\left[\mathcal{F}_{2}\left(\alpha_{2}\right) l_{2}-\mathcal{F}_{1}\left(\alpha_{1}\right) l_{1}\right]+ \\
& +\frac{v}{l_{1}+l_{2}}\left(\alpha_{2}-\alpha_{1}\right)-\frac{1}{m v}\left[\mathcal{F}_{1}\left(\alpha_{1}\right)+\mathcal{F}_{2}\left(\alpha_{2}\right)\right], \\
\mathrm{f}_{2}= & \frac{l_{2}}{J v}\left[\mathcal{F}_{1}\left(\alpha_{1}\right) l_{1}-\mathcal{F}_{2}\left(\alpha_{2}\right) l_{2}\right]+ \\
& +\frac{v}{l_{1}+l_{2}}\left(\alpha_{2}-\alpha_{1}\right)-\frac{1}{m v}\left[\mathcal{F}_{1}\left(\alpha_{1}\right)+\mathcal{F}_{2}\left(\alpha_{2}\right)\right], \\
h_{1}= & \frac{v}{l_{1}+l_{2}}+\nu, h_{2}=\frac{v}{l_{1}+l_{2}}, \\
g_{1}= & -\frac{l_{1}}{J v}, g_{2}=\frac{l_{2}}{J v},
\end{aligned}
$$

the polynomial state-space representation of the system is formulated as follows:

$$
\dot{x}=\left[\begin{array}{l}
\mathrm{f}_{1}\left(\alpha_{1}, \alpha_{2}\right) \\
\mathrm{f}_{2}\left(\alpha_{1}, \alpha_{2}\right)
\end{array}\right]+\left[\begin{array}{l}
g_{1} \\
g_{2}
\end{array}\right] u+\left[\begin{array}{l}
h_{1} \\
h_{2}
\end{array}\right] d
$$

where $u=M_{b r}, d=\delta$.

\section{FUNDAMENTALS OF SOS PROGRAMMING METHOD}

The SOS programming method is suitable to analyze and control nonlinear polynomial systems. Several papers deal with SOS programming, which has been elaborated in the last decade for control purposes. Important theorems in SOS programming, such as the application of Positivstellensatz, were proposed in [9]. In this way the convex optimization methods can be used to find appropriate polynomials of the SOS problem. Sufficient conditions for the solutions to nonlinear control problems, which were formulated in terms of state-dependent Linear Matrix Inequalities (LMI), were proposed by [10]. In the paper the semidefinite programming relaxations based on the SOS decomposition were then used to efficiently solve such inequalities. A local stability analysis of polynomial systems and an iterative computation method for their region of attraction were presented in [11]. Numerical computation problems of convex programming based SOS methods in practical applications were analyzed in [12].

The basic elements of the method are polynomials and SOS as defined below:

Definition 1: A Polynomial $f$ in $n$ variables is a $f$ nite linear combination of the functions $m_{\alpha}(x):=x^{\alpha}=$ $x_{1}^{\alpha_{1}} x_{2}^{\alpha_{2}} \cdots x_{n}^{\alpha_{n}}$ for $\alpha \in \mathbb{Z}_{+}^{n}$, deg $m_{\alpha}=\sum_{i=1}^{n} \alpha_{i}$ :

$$
f:=\sum_{\alpha} c_{\alpha} m_{\alpha}=\sum_{\alpha} c_{\alpha} x^{\alpha}
$$

with $c_{\alpha} \in \mathbb{R}$. Define $\mathbb{R}$ to be the set of all polynomials in $n$ variables. The degree of $f$ is defined as $f:=\max _{\alpha} \operatorname{deg} m_{\alpha}$.

Definition 2: The set of SOS polynomials in $n$ variables is defined as:

$$
\Sigma_{n}:=\left\{p \in \mathcal{R}_{n} \mid p=\sum_{i=1}^{t} f_{i}^{2}, f_{i} \in \mathcal{R}_{n}, i=1, \ldots, t\right\}
$$

A central theorem of SOS programming is Positivstellensatz. By the application of this theorem the set emptiness constraints of an optimization task can be transformed to SOS feasibility problems.

Theorem 1: [Positivstellensatz] Given polynomials $\left\{f_{1}, \ldots, f_{r}\right\},\left\{g_{1}, \ldots, g_{t}\right\}$ and $\left\{h_{1}, \ldots, h_{u}\right\}$ in $\mathcal{R}_{n}$, the following are equivalent:

1) The set

$$
\left\{\begin{array}{l|l}
x \in \mathbb{R}^{n} & \begin{array}{c}
f_{1}(x) \geq 0, \ldots, f_{r}(x) \geq 0 \\
g_{1}(x) \neq 0, \ldots, g_{t}(x) \neq 0 \\
h_{1}(x)=0, \ldots, h_{u}(x)=0
\end{array}
\end{array}\right\}
$$

is empty.

2) There exists polynomials $f \in \mathcal{P}\left(f_{1}, \ldots, f_{r}\right) \quad(\mathcal{P}$ is cone), $g \in \mathcal{M}\left(g_{1}, \ldots, g_{t}\right)$ ( $\mathcal{M}$ is multiplicative monoid), $h \in \mathcal{I}\left(h_{1}, \ldots, h_{u}\right)$ (I is ideal) such that

$$
f+g^{2}+h=0
$$

There is an important connection between SOS programming and LMI problems, which was proved by [9]:

Theorem 2: Given a finite set $\left\{p_{i}\right\}_{i=0}^{m} \in \mathcal{R}_{n}$, the existence of $\left\{a_{i}\right\}_{i=0}^{m} \in \mathbb{R}_{n}$ such that

$$
p_{0}+\sum_{i=1}^{m} a_{i} p_{i} \in \Sigma_{n}
$$

is an LMI feasibility problem.

The previous two theorems can be used to prove the generalization of the S-Procedure, which is highly significant in the forthcoming computations.

Theorem 3: [Generalized S-Procedure] Given symmetric matrices $\left\{p_{i}\right\}_{i=0}^{m} \in \mathcal{R}_{n}$. If there exist nonnegative scalars $\left\{s_{i}\right\}_{i=1}^{m} \in \Sigma_{n}$ such that

$$
p_{0}-\sum_{i=1}^{m} s_{i} p_{i} \succeq q
$$


with $q \in \Sigma_{n}$, then

$$
\bigcap_{i=1}^{m}\left\{x \in \mathbb{R}^{n} \mid p_{i}(x) \geq 0\right\} \subseteq\left\{x \in \mathbb{R}^{n} \mid p_{0}(x) \geq 0\right\}
$$

The related set emptiness question asks if

$$
\begin{array}{r}
W:=\left\{x \in \mathbb{R}^{n} \mid p_{1}(x) \geq 0, \ldots, p_{m}(x) \geq 0,\right. \\
\left.-p_{0}(x) \geq 0, p_{0}(x) \neq 0\right\}
\end{array}
$$

is empty.

\section{Computation Method of Controlled INVARIANT SETS}

The goal of the nonlinear actuator analysis is the determination of its intervention limits besides a peak-bounded actuation. This problem leads to the computation of Controlled Invariant Sets [13].

The state-space representation of the system is given in the following form (see (7)):

$$
\dot{x}=f(x)+g u
$$

where $f(x)$ is a matrix, which incorporates smooth polynomial functions and $f(0)=0$. In the next analysis one control input is considered, thus $u=M_{b r}$ or $u=\delta$. The global asymptotical stability of the system at the origin is guaranteed by the existence of the Control Lyapunov Function of the system defined as follows [14]:

Definition 3: A smooth, proper and positive-definite function $V: \mathbb{R}^{n} \rightarrow \mathbb{R}$ is a Control Lyapunov Function for system if

$$
\inf _{u \in \mathbb{R}}\left\{\frac{\partial V}{\partial x} f(x)+\frac{\partial V}{\partial x} g \cdot u\right\}<0
$$

for each $x \neq 0$.

Two main cases are distinguished:

Case A: If $\frac{\partial V}{\partial x} f(x)<0$ then the system is stable and $u \equiv 0$. This stability scenario is contained by the next two stability criteria.

Case B: If $\frac{\partial V}{\partial x} f(x)>0$ then the system is unstable. However, the system can be stabilized.

Case B/1: If $\frac{\partial V}{\partial x} g<0$ and $\frac{\partial V}{\partial x} f(x)+\frac{\partial V}{\partial x} g \cdot u_{\max }<0$, the upper peak-bound of control input $u$ stabilizes the system.

Case B/2: If $\frac{\partial V}{\partial x} g>0$ and $\frac{\partial V}{\partial x} f(x)-\frac{\partial V}{\partial x} g \cdot u_{\max }<0$, the lower peak-bound of control input $u$ stabilizes the system. Note that $u_{\min }=-u_{\max }$.

The Controlled Invariant Set of the system (16) is defined as the level-set of the Control Lyapunov Function at $V(x)=$ 1. Thus, the fulfilment of the previous stability criterion must be guaranteed at $V(x) \leq 1$.

Moreover, the Positivstellensatz and Generalized SProcedure theorems require greater than or equal $(\geq)$ conditions to formulate SOS conditions. Thus, the condition $\frac{\partial V}{\partial x} g<0$ in Case $\mathrm{B} / 1$ is rewritten to $\frac{\partial V}{\partial x} g \leq-\epsilon$, where $\epsilon \in \mathbb{R}^{+}$is as small as possible. Similarly in Case $B / 2$ $\frac{\partial V}{\partial x} g \geq \epsilon$ is written. Additionally, the conditions $\frac{\partial V}{\partial x} f(x) \pm$ $\frac{\partial V}{\partial x} g \cdot u_{\max }<0$ in Cases $\mathrm{B} / 1$ and $\mathrm{B} / 2$ are also reformulated to two conditions: $\frac{\partial V}{\partial x} f(x) \pm \frac{\partial V}{\partial x} g \cdot u_{\max } \leq 0$ and $\frac{\partial V}{\partial x} f(x) \pm$ $\frac{\partial V}{\partial x} g \cdot u_{\max } \neq 0$.
Above the stability criterion of the polynomial system has been formed. Based on these constraints it is necessary to find a Control Lyapunov Function $V$ which meets the following set emptiness conditions:

$$
\begin{gathered}
\left\{-\frac{\partial V}{\partial x} g-\epsilon \geq 0,1-V(x) \geq 1, l_{1}(x) \neq 0,\right. \\
\frac{\partial V}{\partial x} f(x)+\frac{\partial V}{\partial x} g \cdot u_{\max } \geq 0 \\
\left.\frac{\partial V}{\partial x} f(x)+\frac{\partial V}{\partial x} g \cdot u_{\max } \neq 0\right\}=\emptyset \\
\left\{\frac{\partial V}{\partial x} g-\epsilon \geq 0,1-V(x) \geq 1, l_{2}(x) \neq 0,\right. \\
\frac{\partial V}{\partial x} f(x)-\frac{\partial V}{\partial x} g \cdot u_{\max } \geq 0, \\
\left.\frac{\partial V}{\partial x} f(x)-\frac{\partial V}{\partial x} g \cdot u_{\max } \neq 0\right\}=\emptyset
\end{gathered}
$$

Note that the relations in the third inequality are inverted to guarantee the emptiness of the sets. The role of $l_{1,2}(x) \neq 0$ is to guarantee the condition $x \neq 0$ in (3). $l_{1,2}(x)$ is chosen as a positive definite polynomial [15]. Since it is necessary to find the maximum Controlled Invariant Set, another set emptiness condition is also defined to improve the efficiency of the method [15]:

$$
\{p(x) \leq \beta, V(x) \geq 1, V(x) \neq 1\}=\emptyset
$$

where $p \in \Sigma_{n}$ is a fixed and positive definite function. $\beta$ defines a $P_{\beta}:=\left\{x \in \mathbb{R}^{n} \mid p(x) \leq \beta\right\}$ level set, which is incorporated in the actual Controlled Invariant Set. Thus, the maximization of $\beta$ enlarges $P_{\beta}$ together with the Controlled Invariant Set.

The set emptiness conditions are reformulated to SOS conditions based on the S-procedure (see Section III). Thus, the next optimization problem is formed to find the maximum Controlled Invariant Set:

$$
\max \beta
$$

over $s_{1}, s_{2}, s_{3}, s_{4}, s_{5} \in \Sigma_{n} ; V, p_{1}, p_{2} \in \mathcal{R}_{n} ; V(0)=0$ such that

$$
\begin{aligned}
-\left(\frac{\partial V}{\partial x} f(x)+\frac{\partial V}{\partial x} g \cdot u_{\max }\right) & -s_{1}\left(-\frac{\partial V}{\partial x} g-\epsilon\right)- \\
& -s_{2}(1-V)-p_{1} l_{1} \in \Sigma_{n} \\
-\left(\frac{\partial V}{\partial x} f(x)-\frac{\partial V}{\partial x} g \cdot u_{\max }\right) & -s_{3}\left(\frac{\partial V}{\partial x} g-\epsilon\right)- \\
& -s_{4}(1-V)-p_{2} l_{2} \in \Sigma_{n}
\end{aligned}
$$

$-\left(s_{5}(\beta-p)+(V-1)\right) \in \Sigma_{n}$

The optimization method of the maximum Controlled Invariant Set has been proposed in the previous parts of the section. Although (22) provides an appropriate solution to the optimization problem, it results in numerical difficulties. 
Note that the degree of $f(x)$ is determined by the degree of the lateral tire model, see (3).

In the following an alternative computation method is proposed to find the maximum Controlled Invariant Set, which, in our experience, can lead to an easier calculation. The practical method contains a three-step iterative method.

Step 1: The region of attraction of the uncontrolled system $\dot{x}=f(x)$ is determined as an initial set. In this step the maximum level set of $V_{0}=1$ is found, which is incorporated in the stable region. The SOS based computation of the region of attraction is presented in [16].

Step 2: An $\eta$ parameter is chosen and $V_{\eta}=V_{0} \cdot \eta$ is checked as a Local Control Lyapunov Function. The levelset $V_{\eta}=1$ represents a Controlled Invariant Set $S_{\eta}$, in which the system can be stabilized using a finite control input $u$. Depending on parameter $\eta$ the size of the level-set can be enlarged or reduced. The SOS based computation of Local Control Lyapunov Function is proposed in [11].

Step 3: In the final step the acceptability and the enlarging possibility of $S_{\eta}$ Controlled Invariant Set must be checked. The peak-bounds of the actuation are $u_{\min }=-u_{\max }$ and $u_{\text {max }}$. $S_{\text {inst }}=\frac{\partial V}{\partial x} f(x)>0$ is the instable region of the system. $S_{\min }=\frac{\partial V}{\partial x} f(x)-\frac{\partial V}{\partial x} g \cdot u_{\max }>0$ is the region, which can not be stabilized by $u_{\min }$. Similarly, $S_{\max }=$ $\frac{\partial V}{\partial x} f(x)+\frac{\partial V}{\partial x} g \cdot u_{\max }>0$ is the region, which can not be stabilized by $u_{\max }$. If $S_{\eta}$ is an appropriate Controlled Invariant Set and $V_{\eta}$ is an appropriate Control Lyapunov Function, then

$$
S_{\eta} \bigcap S_{\text {inst }} \bigcap S_{\min } \bigcap S_{\max }=\emptyset
$$

The emptiness of the intersection condition defined below can be checked manually by the plot of $S_{\eta}, S_{\text {inst }}, S_{\min }$ and $S_{\max }$. Additionally, if $S_{\eta}$ is appropriate then $\eta$ value can be reduced in the previous step to maximize the Controlled Invariant Set.

\section{Control strategies using Controlled INVARIANT SETS}

In this section the control strategy based on the maximum Controlled Invariant Sets is presented. The aim of the control strategy is to guarantee the lateral stability of the vehicle, while differential braking is assisting the driver.

The control problem is the following. The driver of the vehicle performs a steering maneuver to turn the vehicle. Based on the steering angle $\delta$ and velocity $v$ the reference yaw-rate $\dot{\psi}_{\text {ref }}$ required by the driver is computed [17]. The aim of the lateral control system is to guarantee the tracking of $\dot{\psi}_{\text {ref }}$ using an appropriate peak-bounded $M_{b r}$ signal. Thus it is necessary to design a control strategy which guarantees:

$$
\left|\dot{\psi}_{\text {ref }}-\dot{\psi}\right| \rightarrow \min \quad \text { such that } \quad M_{b r} \leq M_{b r, \max }
$$

Moreover, the designed control strategy must handle the nonlinearities of the tire to guarantee the stability of the vehicle in an extended region. A further requirement is to minimize the computational effort of the algorithm because of the real-time implementation.
In the following a yaw-rate tracking control strategy based on the combination of maximum Controlled Invariant Sets and LQ control technique is proposed. In this solution the linear and nonlinear regions of the vehicle dynamics are separated. In the linear region $\left(|\alpha| \leq 4^{\circ}\right)$ the aim of the control system is to track $\dot{\psi}_{\text {ref }}$. Beyond it the nonlinear characteristics of the tire are dominant $\left(4^{\circ}<|\alpha|<12^{\circ}\right)$, thus the instability of the vehicle must be avoided using an appropriate control input signal.

\section{A. Control strategy in the linear region}

In the linear region the LQ control strategy is applied to guarantee yaw-rate tracking with constrained control input (26). In the linear tire region the control is designed based on the bicycle model with linear tire characteristics. The system is transformed into a state-space form, such as:

$$
\begin{aligned}
\dot{x} & =A x+B M_{b r} \\
z & =C_{1} x+D_{11} \dot{\psi}+D_{12} M_{b r} \\
y & =C_{2} x
\end{aligned}
$$

where $x=\left[\begin{array}{ll}\dot{\psi} & \beta\end{array}\right]^{T}$, the performance vector contains $z=$ $\left[\begin{array}{ll}\dot{\psi}_{r e f}-\dot{\psi} & M_{b r}\end{array}\right]^{T}$ and the measured signals $y=\left[\begin{array}{ll}\dot{\psi} & a_{y}\end{array}\right]^{T}$ are the yaw rate and lateral acceleration.

The aim of the optimal control based on the Linear Quadratic (LQ) criterion is to minimize the performances of the system using the following cost function:

$$
J=\frac{1}{2} \int_{0}^{\infty}\left[Q \cdot\left(\dot{\psi}_{\text {ref }}-\dot{\psi}\right)^{2}+R \cdot M_{b r}^{2}\right] d t \rightarrow \min
$$

where $Q$ and $R$ are design parameters. Increasing $Q$ results in an improvement of the tracking control. However, the consequence of a high $Q$ value is an increased $M_{b r}$. The result of the optimization is a controller $K_{2 \times 1}$, which is used for the computation of the control input:

$$
M_{b r}=K_{1}\left(\dot{\psi}_{r e f}-\dot{\psi}\right)-K_{2} \beta
$$

The designed optimal control guarantees a balance between the performances by using weighting functions $Q$ and $R$. However, it is also important to consider the limitation of the control input (26). Avoiding actuator saturation is ensured by the appropriate selection of the weighting function $R$.

The limitation of the control input can be considered by the appropriate selection of the weights. A possible way to avoid the saturation of $M_{b r}$ and guarantee accurate tracking is switching between $K_{i}, i=1 \ldots n$ controllers, which are designed using different weights [18]. A designed $K_{i}$ controller is able to guarantee $\left|M_{b r}\right| \leq M_{b r, \max }$, if $M_{b r, \max } \leq\left|K_{1}\left(\dot{\psi}_{\text {ref }}-\dot{\psi}\right)-K_{2} \beta\right|$, see (31). When state vector $x$ is transformed into tire side-slip $\alpha_{1,2}$ values, the $K_{i}$ validity region is: 


$$
\begin{aligned}
\alpha_{2} \geq & \frac{K_{i, 1} v+K_{i, 2} l_{2}}{K_{i, 1} v-l_{1} K_{i, 2}} \alpha_{1}+\frac{K_{i, 1}\left(l_{1}+l_{2}\right)}{K_{i, 1} v-l_{1} K_{i, 2}} \dot{\psi}_{r e f} \\
& -\frac{l_{1}+l_{2}}{K_{i, 1} v-l_{1} K_{i, 2}} M_{b r, \max } \\
\alpha_{2} \leq & \frac{K_{i, 1} v+K_{i, 2} l_{2}}{K_{i, 1} v-l_{1} K_{i, 2}} \alpha_{1}+\frac{K_{i, 1}\left(l_{1}+l_{2}\right)}{K_{i, 1} v-l_{1} K_{i, 2}} \dot{\psi}_{r e f} \\
& +\frac{l_{1}+l_{2}}{K_{i, 1} v-l_{1} K_{i, 2}} M_{b r, \max } \\
\left|\alpha_{1}\right| & \leq 4^{\circ} \\
\left|\alpha_{2}\right| & \leq 4^{\circ}
\end{aligned}
$$

The validity regions of all $K_{i}$ are illustrated in Figure 2. In the control strategy several controllers are applied with different $Q, R$ weights. By monitoring the side-slip angles the appropriate $K_{i}$ belonging to $\alpha_{1}, \alpha_{2}$ is chosen. The

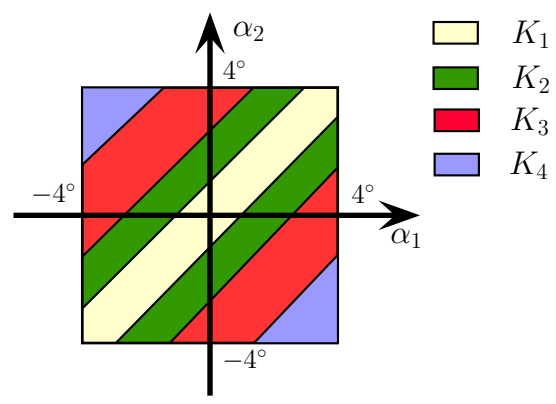

Fig. 2. Sets of validity regions $\left(\dot{\psi}_{\text {ref }}=0\right)$

switching strategy in the linear region guarantees not only the satisfaction of the input constraint, but also the yaw-rate tracking performance. Note that in Figure 2 the controller $K_{1}$ ensures the most accurate tracking but it has a tight validity region. For higher side-slip values a more conservative $K_{4}$ controller is required. $K_{4}$ has a higher tracking error, but it consumes less control input.

\section{B. Control strategy in the nonlinear region}

The goal of the control strategy in the nonlinear tire region $\left(4^{\circ}<|\alpha|<12^{\circ}\right)$ is to guarantee lateral stability. If the tire side-slip angle is out of the linear region, the control strategy must take back the states into the linear region, in which the switching LQ strategy guarantees the yaw-rate tracking.

The control strategy in the nonlinear region is based on the maximum Controlled Invariant Set analysis. In Section IV the effects of the maximum/minimum control inputs on the lateral stability were investigated. During the analysis it was proved that inside the maximum Controlled Invariant Set $\pm M_{b r, \max }$ is able to stabilize the system. Therefore different regions are defined inside the maximum Controlled Invariant Set, such as:

$$
\begin{aligned}
& S_{\text {max }}=\left\{x=\left[\alpha_{1}, \alpha_{2}\right]^{T} \in \mathbb{R}^{n} \mid \frac{\partial V}{\partial x} f(x)-\frac{\partial V}{\partial x} M_{b r, \text { max }}<0\right\} \\
& S_{\text {min }}=\left\{x=\left[\alpha_{1}, \alpha_{2}\right]^{T} \in \mathbb{R}^{n} \mid \frac{\partial V}{\partial x} f(x)+\frac{\partial V}{\partial x} M_{b r, \text { max }}<0\right\}
\end{aligned}
$$

In region $S_{\max }$ the control input is $u \equiv M_{b r, \max }$, while in $S_{\min }$ region $u \equiv-M_{b r, \max }$. It means that the maximum Controlled Invariant Set-based strategy results in a bangbang controller. Note that the regions $S_{\max }, S_{\min }$ can be computed offline, since they do not depend on the actual states.

The state-space $\alpha_{1}, \alpha_{2}$ is divided into several regions based on the linear control strategy and the maximum Controlled Invariant Set, see Figure 3. The implementation of the control algorithm is based on the offline computation of the regions and the monitoring of the actual tire side-slips. The control input computation algorithms of each region are relatively simple, see (31) and (36), which facilitate the controller implementation.

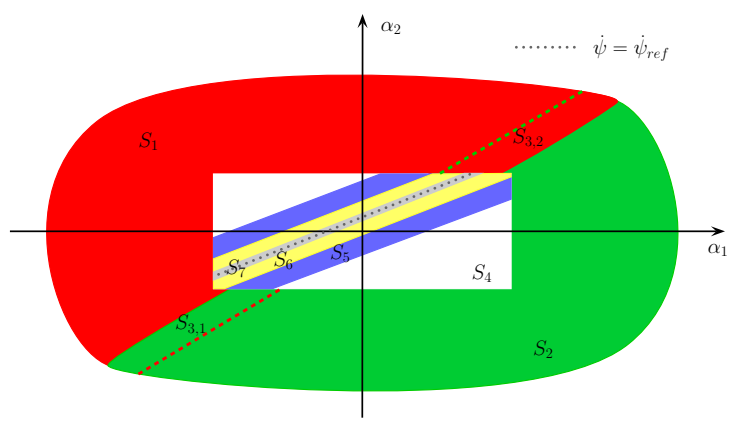

Fig. 3. Sets of different controllers

Note that in several control applications the switching causes chattering. In this control strategy chattering can occur when the state trajectory passes through the boundary of $S_{\max }$ and $S_{\min }$. Thus, hysteretic areas between the regions $S_{3,1}$ and $S_{3,2}$ are applied to avoid chattering.

It is necessary to consider that the presented control algorithm requires the estimation of $\alpha_{1}, \alpha_{2}$ signals and their transformation into $\dot{\psi}, \beta$ for LQ control. However, in practice the yaw-rate and the lateral acceleration signals are measured. Estimation methods of $\beta$ can be found in [19]. Based on the estimated vehicle side slip angle the tire side slip angles are calculated.

\section{Demonstration EXAMPLE}

In this section the operation of the control strategy is demonstrated through an example. The aim of the control is to guarantee the stability and the yaw-rate tracking of the vehicle. In the proposed scenario the lateral tire side-slip of the vehicle $\alpha_{2}$ has a high value $\left(\alpha_{2}=9^{\circ}\right)$ and the vehicle is inside of an unstable region. However, the vehicle can be stabilized using an appropriate control input $M_{b r}$. 
The state trajectory of the system is found in Figure 4. The tire side-slips of the vehicle are reduced during the actuation, therefore the trajectory is moved into the linear region, see Figure 4(a). Inside the square the trajectory passes through several controller regions, see Figure 4(b).

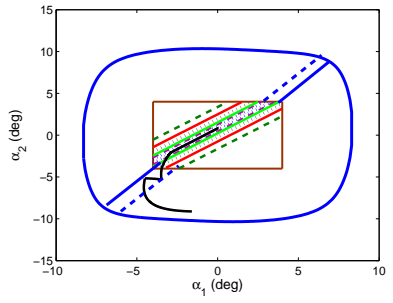

(a) Entire region

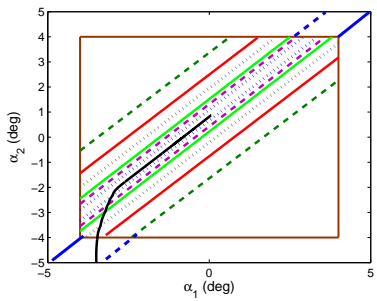

(b) Linear region
Fig. 4. Slip trajectory of the vehicle

The illustration of the yaw-rate tracking capability of the controlled system is found in Figure 5(a). The designed control system is able to guarantee the tracking of $\dot{\psi}_{r e f}$ and handle the nonlinear behavior of the system. The reduction of $\alpha_{1}, \alpha_{2}$ angles also has an effect on $\beta$, see Figure 5(b). Decreasing vehicle side-slip improves the lateral stability of the system. Figure 5(c) shows the control input of the system. It can be stated that the control input limitation $M_{b r, \max }=$ $15000 \mathrm{Nm}$ is satisfied by the proposed algorithm. The sharp change of $M_{b r}$ at $10 \mathrm{sec}$ is the crossing of the border of hysteretic area $S_{3,1}$. At $15 \mathrm{sec}$ the vehicle trajectory crosses the borders inside the linear region, see Figure 4(b). The switching sequence is found in Figure 5(d). Number 0 means that the maximum Control Lyapunov Function-based rule is activated (36). Numbers $1 \ldots 5$ are related to the linear controllers, where controller 5 is the most conservative with the smallest $Q$ gain. The frequent switches between 13...15s are inside the linear region. The numerous controllers provide as much as possible control input with the consideration of $M_{b r, \max }$ and the improvement of yaw-rate tracking.

\section{CONCLUSION}

In the paper the reachability characteristics of the brake system have been examined in order to analyse its abilities for the entire vehicle system. A nonlinear polynomial SOS programming method is applied to calculate the shape of the reachable sets of actuators. The aim of the analysis is to provide a theoretical basis for the coordination of the actuators. As the simulation example has shown the proposed control algorithm is able to guarantee the stabilization of the lateral dynamics and the tracking of a yaw-rate signal.

\section{REFERENCES}

[1] S. Sadri and C. Wu, "Stability analysis of a nonlinear vehicle model in plane motion using the concept of Lyapunov exponents," Vehicle System Dynamics, vol. 51, no. 6, pp. 906-924, 2013.

[2] G. Palmieri, M. Baríc, L. Glielmo, E. H. Tseng, and F. Borrelli, "Robust vehicle lateral stabilization via set-based methods for uncertain piecewise affine systems: Experimental results," 50th IEEE Conference on Decision and Control, Orlando, USA, pp. 3252-3257, 2011.

[3] A. Carvalho, G. Palmieri, E. H. Tseng, L. Glielmo, and F. Borrelli, "Robust vehicle stability control with an uncertain driver model," IEEE European Control Conference, Zurich, Switzerland, 2013.

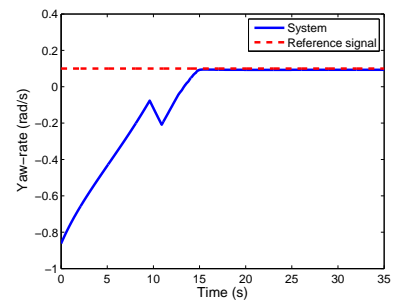

(a) Yaw-rate tracking

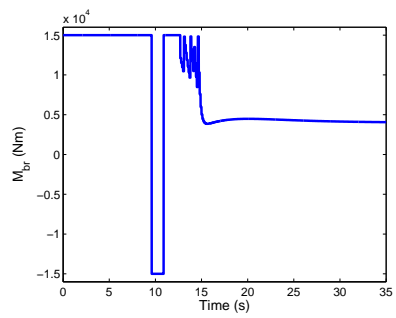

(c) Control input

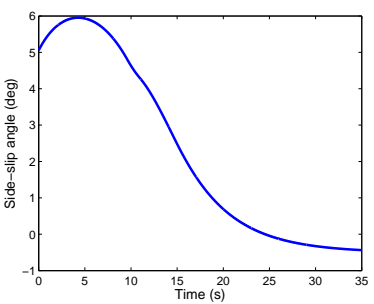

(b) Vehicle side-slip

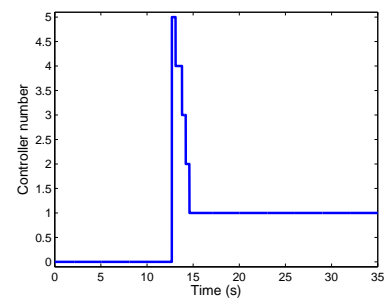

(d) Selected controller
Fig. 5. Operation of the controlled system

[4] K. Kritayakirana and J. C. Gerdes, "Autonomous vehicle control at the limits of handling," International Journal of Vehicle Autonomous Systems, vol. 10, no. 4, pp. 271-296, 2012.

[5] C. E. Beal and J. C. Gerdes, "Model predictive control for vehicle stabilization at the limits of handling," IEEE Transactions on Control Systems Technology, vol. 21, no. 4, pp. 1258-1269, 2013.

[6] B. Németh and P. Gáspár, "Analysis of vehicle actuators based on reachable sets," European Control Conference, Zurich, Switzerland, 2013.

[7] U. Kiencke and L. Nielsen, Automotive control systems for engine, driveline and vehicle. Springer, 2000.

[8] C. C. de Wit, H. Olsson, K. J. Astrom, and P. Lischinsky, "A new model for control of systems with friction," IEEE Transactions on Automatic Control, vol. 40, no. 3, pp. 419-425, 1995.

[9] P. Parrilo, "Semidefinite programming relaxations for semialgebraic problems," Mathematical Programming Ser. B, vol. 96, no. 2, pp. 293320, 2003.

[10] S. Prajna, A. Papachristodoulou, and F. Wu., "Nonlinear control synthesis by sum of squares optimization: A Lyapunov-based approach," 5th IEEE Asian Control Conference, vol. 1, pp. 157-165, 2004.

[11] W. Tan and A. Packard, "Stability region analysis using polynomial and composite polynomial lyapunov functions and sum-of-squares programming," IEEE Transactions on Automatic Control, vol. 53, no. 2, pp. 565-571, 2008.

[12] J. Löfberg, "Pre- and post-processing Sum-of-Squares Programs in practice," IEEE Transactions on Automatic Control, vol. 54, no. 5, pp. 1007-1011, 2009.

[13] M. Korda, D. Henrion, and C. N. Jones, "Convex computation of the maximum controlled invariant set for polynomial control systems," 2013.

[14] E. D. Sontag, "A "universal" construction of Artstein's Theorem on nonlinear stabilization," Systems \& Control Letters, vol. 13, pp. 117$123,1989$.

[15] Z. Jarvis-Wloszek, R. Feeley, W. Tan, K. Sun, and A. Packard, "Some controls applications of sum of squares programming," 42nd IEEE Conference on Decision and Control, Maui, USA, vol. 5, pp. 46764681, 2003.

[16] Z. Jarvis-Wloszek, Lyapunov Based Analysis and Controller Synthesis for Polynomial Systems using Sum-of-Squares Optimization. Berkeley: Ph.D. Thesis, University of California, 2003.

[17] R. Rajamani, "Vehicle dynamics and control," Springer, 2005.

[18] G. F. Wredenhagen and P. R. Bélanger, "Piecewise-linear LQ control for systems with input constraints," Automatica, vol. 30, pp. 403-416, 1994.

[19] H. F. Grip, L. Imsland, T. A. Johansen, T. I. Fossen, J. C. Kalkkuhl, and A. Suissa, "Nonlinear vehicle side-slip estimation with friction adaptation," Automatica, vol. 44, pp. 611-622, 2008. 